AIAA-2003-1829

\title{
AN ARTICULATED MOTION AND VIBRATION ISOLATOR FOR SPACECRAFT
}

\author{
Thomas W. Murphey* \\ Matthew Botke ${ }^{\dagger}$ \\ Ross M. Blankinship \\ Capt. Nick T. Hague ${ }^{\S}$
}

\begin{abstract}
A novel concept is presented for motion and vibration isolation of a spacecraft from its subsystems. The isolator is an articulated chain of six rolamite joints arranged to give the spacecraft and subsystems full relative mobility (three translational and three rotational degrees of freedom). This mobility decouples spacecraft and subsystem motions, enabling agile missions where the system bus and subsystems slew independently. The rolamite joints freely rotate with low friction so that the system also behaves as a vibration isolator. Motions of one end of the chain are conveyed to the other only through reaction forces (d'Alembert forces) caused by acceleration of the isolator links. The inverse kinematic and dynamic equations of motion of the isolator are derived and solved for a reference isolator design. Numerical results that quantify the design's vibration isolation characteristics are presented. The studies estimate that a $0.02 \mathrm{~Hz}$ harmonic displacement at one end of the isolator translates to a reaction force on the other end of $0.003 \mathrm{~N}$ per $m$ (17e-6 lb/in) of input vibration amplitude over large operational ranges.
\end{abstract}

\section{Introduction}

Spacecraft are often required to slew or slew subsystems in an effort to point at distant objects. The pointing requirements that follow from these maneuvers broadly fall into the category of either jitter (harmonic vibration type pointing errors) or agility (the quickness, accuracy and range over which maneuvers can be performed). Complicating the matter, however,

\footnotetext{
${ }^{*}$ Research Associate, University of Cambridge, England, Member AIAA

'Senior Engineer, AEC-Able Engineering, Inc., Goleta, CA

${ }^{\ddagger}$ Associate Principal Engineer, CSA Engineering, Inc., Albuquerque, NM

${ }^{\S}$ Space Concepts Engineer, Air Force Research Lab, Albuquerque, NM, Member AIAA

Copyright (C) 2003 by Thomas W. Murphey. Published by the American Institute of Aeronautics, Inc. with permission.
}

different subsystems perform vastly different tasks and are subjected to correspondingly different requirements. For example, solar arrays have lenient requirements while optical instruments often have very stringent requirements. For many spacecraft it is reasonable to follow the approach of subjecting all components to the most stringent requirements of any of the components. When this comes at a great cost it may no longer be an efficient philosophy to follow. Isolation techniques are then required to separate the influences of various subsystems. Such an isolation technique is the topic of this paper.

Structurally, jitter requirements manifest as a minimum frequency requirement on the first mode of system vibration. This requirement drives many aspects of a structure as it dictates the stiffness and structural depth of the structure. Thus, frequency provides a basis for estimating the structural cost associated with not isolating subsystems. A simple analysis is now used to evaluate this cost.

Several subsystems (solar arrays, instruments on booms, etc.) may be represented by an analytical model of a cantilever beam that deforms only through bending. Assume a sandwich panel type beam with cross sectional area moment of inertia given by,

$$
I=\frac{1}{4} A d^{2}
$$

where $A$ is the combined cross sectional area of both face sheets and $d$ is the distance between face sheets. The fundamental vibration frequency $\left(f_{\text {cant }}, \mathrm{Hz}\right)$ of such a beam is given by,

$$
f_{\text {cant }}=\frac{0.280 d}{l^{2}} \sqrt{\frac{E}{\rho}}
$$

where $\rho$ is the face sheet mass density. ${ }^{1}$ Consider the dynamic behavior of this beam as the beam length $(l)$ is scaled by the factor $s$,

$$
l^{\prime}=s l
$$

To maintain constant frequency, the scaled structure depth $\left(d^{\prime}\right)$ must scale as the square of the scale factor,

$$
d^{\prime}=s^{2} d
$$


In other words, if the length of the structure is doubled $(s=2)$, its depth must be increased by a factor of $s^{2}=4$.

It is challenging to design space structures that are deep and also package well for launching. While a detailed study has not been performed here, there appears to be a correlation between deployed structure depth and packaged structure volume. ${ }^{2}$ An obvious option to enable larger and more packageable structures is to design to less stringent pointing requirements which are derived only from the subsystem's function perspective. Such is the premise of the SquareRigger, a scalable, high performance deployable solar array., ${ }^{3,4}$ In larger configurations, SquareRigger is intended to have a fundamental frequency of only $0.02 \mathrm{~Hz}$. This approach, however, requires an isolator to mitigate the disturbances caused by a low frequency array. This challenge has motivated the development of a new vibration isolator concept. The concept, referred to as the Traveler-ARM (Transient Vibration Eliminating Elastic Rolamite Articulated Reactionless Mooring System), is presented and dynamically characterized in this paper.

Before introducing the details of the TravelerARM, more insight can be advanced from the simple beam model by considering how system bus jitter error scales with beam length. In this analysis, vibration energy (as opposed to frequency in the previous analysis) will be held constant. Assume the root of the cantilever beam is attached to a much more massive system bus. Vibration of the beam transmits a harmonic torque of amplitude $T_{\max }$ and frequency $f_{\text {cant }}$ to the bus, which has rotational inertia $I_{\text {bus }}$. The amplitude of the resulting harmonic bus rotations (jitter error) is given by,

$$
\theta_{\max }=\frac{T_{\max }}{I_{\text {bus }}\left(2 \pi f_{\text {cant }}\right)^{2}}
$$

Equation (5) shows the strong dependence of jitter on frequency; halving the cantilever mode frequency increases the jitter error by a factor of 4 .

The harmonic root reaction torque from a vibrating cantilever beam of bending stiffness $E I$ has amplitude given by,

$$
T_{\max }=3.516 \frac{E I}{l^{2}} y_{\max }
$$

where $y_{\max }$ is the amplitude of vibration measured at the beam tip. The amplitude of vibration as a function of vibration mode energy content $\left(U_{\text {cant }}\right)$ is given by, ${ }^{5}$

$$
y_{\max }=0.804 \sqrt{\frac{U_{\text {cant }} l^{3}}{E I}}
$$

Substitution of Equation (7) into Equation (6) and the result into Equation (5) gives the system bus jitter error due to a cantilever beam vibrating with frequency, $f_{\text {cant }}$, and energy, $U_{\text {cant }}$,

$$
\theta_{\max }=\frac{2.828}{I_{\text {bus }}\left(2 \pi f_{\text {cant }}\right)^{2}} \sqrt{\frac{U_{\text {cant }} E I}{l}}
$$

As a final step, the cantilever beam vibration frequency given by Equation (2) is substituted into Equation (8) to yield,

$$
\theta_{\max }=\frac{0.458 \rho}{d I_{b u s}} \sqrt{\frac{A U_{\text {cant }} l^{7}}{E}}
$$

For fixed appendage depth and vibration energy content, the bus jitter error scales with appendage length to the $7 / 2$ power. Within the assumptions of this analysis, if the appendage length is doubled, the system bus jitter error increases by a factor of 11.3. To prevent jitter error from increasing would require increasing the structure depth by the same factor of 11.3. In precision applications, these results drive the depth requirement of non-isolated structures to levels that are feasible, but come at the consequence of increased design challenge and packaged structure volume.

\section{Traveler-ARM Premise}

An approach to prevent jitter is to remove the stiffness path through which vibrations transmit disturbances. While to the author's knowledge this approach has not been applied to space systems, it is not new. It is employed by the Steadicam (Figure 1) line of camera stabilization devices invented by Garret Brown. ${ }^{6,7}$ These devices maintain articulated mechanical connections to instruments, yet only minimally influence their dynamics. They accomplish this through very low spring rate mechanisms that are preloaded to compensate for gravity (in space, the springs and preload are unnecessary). In theory, the only significant force transmissions from these devices come from the dynamic mass (inertia) of the device. These reaction forces are referred to as d'Alembert forces and are proportional to the acceleration of the device.

The isolation characteristics of these articulated isolators are quite contrary to traditional passive springmass-damper (SMD) isolators. SMD systems do not isolate at frequencies below their fundamental frequency (input equals output for DC input). For fixed amplitude input displacement, increasing frequency to well above the natural frequency reduces the output displacement amplitude and the SMD system behaves as an isolator.

In contrast, articulated isolators create disturbing forces only to the extent that their effective mass $\left(m_{\text {eff }}\right)$ must be accelerated, 


$$
F=m_{\text {eff }} a
$$

The effective mass is of the same order as the actual isolator mass, but is potentially much lower. This is because the entire isolator is not accelerated by $a$. A lower acceleration $(a)$ results in a proportionately lower disturbing force $(F)$.

Similar to the fixed appendage configuration, much can be advanced from a simple articulated isolator system model. The model is shown schematically in Figure 2. A point with harmonic motion of amplitude $y_{\text {max }, \text { free }}$ experiences a maximum acceleration of,

$$
a_{\max }=\left(2 \pi f_{\text {free }}\right)^{2} y_{\text {max }, \text { free }}
$$

where the subscript free indicates the change in boundary condition from fixed to free for articulated isolators. A lower subsystem natural frequency results in smaller accelerations and reaction forces. However, by Equation (5), a lower frequency gives the force more time to move the system bus. Because of this contradiction, it is not obvious that a low frequency appendage can effectively be isolated with an articulated isolator.

It is now shown that jitter error is independent of frequency. Assuming the disturbing force is offset from the bus center of gravity by $r$, the resulting disturbing torque on the system bus is given by,

$$
T_{\max }=F_{\max } r=m_{\text {eff }} a_{\text {max }} r=m_{\text {eff }}(2 \pi f)^{2} y_{\text {max }, \text { free }} r
$$

This equation indicates the articulate isolator reaction forces scale with frequency squared. Substitution of Equation (12) into Equation (5) gives,

$$
\theta_{\max , \text { free }}=\frac{m_{e f f}(2 \pi f)^{2} y_{\text {max }, \text { free }} r}{I_{\text {bus }}(2 \pi f)^{2}}=\frac{m_{e f f} y_{\max , \text { free }} r}{I_{\text {bus }}}
$$

Independent of frequency, the system bus jitter error is a linear function of isolation system mass and appendage vibration amplitude.

Equation (7) is not valid for the current case (it was derived for a cantilever beam). However, a procedure similar to that followed in deriving Equation (7) can be followed for beam free vibration. The fundamental vibration frequency of a free-free beam is given by,

$$
f_{\text {free }}=\frac{1.780 d}{l^{2}} \sqrt{\frac{E}{\rho}}
$$

Paralleling Equation (7), the beam free vibration tip displacement amplitude as a function of mode vibration energy content is,

$$
y_{\max , \text { free }}=0.126 \sqrt{\frac{U l^{3}}{E I}}
$$

Paralleling Equation (9), substitution of Equations (1) and (15) into Equation (13) yields the system bus jitter error as function of vibration energy,

$$
\theta_{\text {max }, \text { rree }}=0.253 \frac{m_{\text {eff }} r}{d I_{\text {bus }}} \sqrt{\frac{U l^{3}}{A E}}
$$

The system bus jitter error resulting from a articulated isolation system scales with structure length to the $3 / 2$ power, significantly less than the $7 / 2$ power relation obtained for a rigidly attached array. If the articulation isolated appendage length is doubled, the system bus jitter error increases by a factor of 2.8 (as compared to 11.3 for a non-isolated appendage). Similarly, to keep jitter error constant the structure depth would need to be increased by the same factor of 2.8 (as compared to 11.3 for a non-isolated appendage).

\section{Traveler-ARM Concept}

While the Traveler-ARM concept is suitable for isolation of a broad range of structures, focus has been placed on implementation in a satellite with two symmetric SquareRigger solar arrays (Figure 3). Figure 4 shows the revolute joint locations and orientations for a reference Traveler-ARM design allowing six degrees of mobility. Essentially, the end joints act as two axis gimbals and the center joint allows the distance between the gimbals to change. The sixth joint (not shown in Figure 4) is equivalent to a solar array alpha joint, requiring continuous rotation. This joint requires two revolutes. The first is a limited rotation rolamite that provides vibration isolation and the second is a displacement driven continuous rotation joint.

The Traveler-ARM decouples SquareRigger and system bus motions and isolates vibrations (within its range of motion). For example, arrays may remain sunpointing as the bus is slewed. Since the bus does not need to move the arrays, it can be highly agile without excessively large actuators. Due to the free motion of the joints, the Traveler-ARM is also performing as a vibration isolator during stationary and slewing operations.

At this point the fundamental mechanics through which the Traveler-ARM isolates vibrations and enables agility are hopefully clear, though unquantified. A fully functional Traveler-ARM is, however, subjected to additional requirements that are critical to the concept. In describing the Traveler-ARM operational philosophy, these additional requirements will become apparent.

\section{Operational Philosophy}

Various perturbations (gravity gradient, solar radiation pressure, atmospheric drag, etc.) cause differential accelerations between the arrays and system bus. Forces must be transmitted through the TravelerARM joints to keep the arrays nominally positioned and oriented relative to the bus. These loads must be transmitted through the Traveler-ARM and somewhat 
disturb the bus. However, the disturbances are of sufficiently low frequency (on the order of the orbit frequency) that reasonable bus attitude control systems are assumed to be sufficient to mitigate them. Bus components required to dump the momentum buildup from secular disturbances must be sized to accommodate these influences, as they would be regardless of the isolation/attachment method. Such influences are impossible to mitigate without an additional set of actuators placed directly on the arrays. While it is conceivable that the position mobility of the Traveler-ARM could be used to nullify certain disturbances, this has not been investigated here.

For these reasons, the Traveler-ARM joints must also serve as force controlled actuators. The forces are quite small, being only those required to counteract solar radiation pressure and gravity gradient. Force control is a key aspect of the joints. Traditional displacement controlled joints would provide a stiffness path that transmits vibrations. Force controlled actuators allow for motion without a change in force; they have zero stiffness.

It is also conceivable that the joints could be actuated in a way that assumes knowledge of a bus slew maneuver. In this way, the joint actuators could be used to move the Traveler-ARM such that the bus doesn't see the added inertia of the Traveler-ARM. Considering that an agile bus would have actuators sized to rotate the bus (which is much more massive than the Traveler-ARM), it is unlikely that the bus will be significantly impacted by the Traveler-ARM inertia. Therefore, such sophisticated joint actuation is not likely to be required.

The specific application of a solar array isolator incurs two additional significant requirements. First, the Traveler-ARM joints must carry significant harnessing, yet remain low friction. Second, the normally free joints must be lockable so that during a system malfunction, the arrays are prevented from causing system damage due to uncontrolled motion (safe mode locking). Umbilical and very soft spring isolators have been proposed for the SquareRigger. These concepts do not easily accommodate safe mode locking. In contrast, the mechanical joints of an articulated isolator are very amenable to locking.

\section{Actuated Conductive Rolamite Joints}

A joint based on conductive rolamite hinges has been identified as meeting the unique requirements of the Traveler-ARM. Figure 5 shows the details of a reference rolamite joint designed by AEC-Able Engineering. Rolamite joints are held together and synchronized through elastic tapes. ${ }^{8}$ The tape's natural (stress-free) configuration is straight; the tapes are elastically rolled around the cylinder. Equal numbers of tapes are wrapped in opposing directions so that joints are elastically balanced; every joint position is a neutrally stable and zero stiffness position. Rolamite joints have no sliding contacts (only rolling) and are therefore, low friction. In this respect, rolamite joints in themselves are not necessarily of lower friction than ball bearings. They are employed here because their synchronization tapes can be made conductive without added friction. Essentially, the joints carry harnessing without added friction.

Preliminary radiation calculations based on beryllium copper alloy (C17410 TH04) were used to size the joint to carry $50 \mathrm{KW}$ at $270 \mathrm{~V}$. This alloy has an electrical conductivity of $45-60 \%$ IACS and a fatigue based elastic strain limit of $0.2-0.23 \%{ }^{9}$ Other beryllium copper alloys allow higher strains, but they have reduced electrical conductivities. The results indicate that a $30 \mathrm{~cm}$ (12 in) wide rolamite joint can carry the required power with less than a $100{ }^{\circ} \mathrm{C}$ temperature rise.

Figure 5 also shows a concept for force control of the joints. A crank arm connects the centers of each rolamite half. One side employs a force controlled motor to apply a torque to the joint. The other side is free to rotate. The position of the crank arm or motor may also serve as a rotational position sensor.

Detailed joint design has not been performed. The model shown is Figure 5 was generated so that a reasonably accurate prediction of the joint mass could be made. The model indicates the described joint will have a total mass of $1.18 \mathrm{~kg}(0.82 \mathrm{~kg}$ for the motor side and $0.36 \mathrm{~kg}$ for the free side for an average mass of 0.6 $\mathrm{kg}(1.32 \mathrm{lb})$ per half).

\section{Traveler-ARM Dynamic Characterization}

The remainder of this paper characterizes the vibration isolation performance of the Traveler-ARM. While the chosen problem formulation allows it, agility is not considered here for the reasons previously discussed. In the analysis, linearized Traveler-ARM dynamic reaction forces per input displacement transfer functions are calculated. These functions predict the amplitude of the harmonic reaction forces seen by the system bus due to harmonic vibrations (displacements) of the appendage (at the appendage / Traveler-ARM interface).

The transfer functions are calculated in a three step analysis. First, kinematic equations describing the motion of the system are derived and second, they are solved for a harmonic input displacement and fixed system bus. In the literature, this process is under the subject of spatial mechanisms and the analysis is called chain inverse kinematic analysis. ${ }^{10}$ The input represents a vibrating structure attached to a system bus (through a Traveler-ARM) that is much more massive 
than the Traveler-ARM. Lastly, the resulting position time histories are used to solve for the dynamic reaction force time histories at joint interfaces. The results are linearized as transfer functions by calculating the ratio of input displacement amplitude to output reaction force amplitude for a diminishingly small input.

If not for the unique kinematic characteristics of rolamite joints, the Traveler-ARM inverse kinematics would be straightforward. However, rolamite joints are kinematically unique in that they do not have a fixed center of rotation as revolute joints do. Rather, rolamites behave as two synchronized parallel axis revolutes in series. This type of joint is often referred to as a gear pair or rolling contact joint.

There are several possible ways to formulate valid kinematic equations for rolamite chains. It was found that many of these allow the correct solution as well as several physically impossible solutions. Many formulations also result in more equations than there are unknowns so that either a subset of the equations is solved or less efficient minimization strategies are required. The approach taken here is to formulate twodimensional kinematic equations that will extend to three-dimensions with minimal modification. While only two-dimensional results are presented, they characterize the dominant motions of the TravelerARM and form a useful reference for further threedimensional studies.

Figure 6 shows the parameters and solution variables in a two-dimensional Traveler-ARM. The length of each chain link (from rolamite half-center to half-center) must remain constant, resulting in five length equations,

$$
\begin{aligned}
& |\overline{b c}|-D=0 \\
& |\overline{c d}|-L=0 \\
& |\overline{d e}|-D=0 \\
& |\overline{e f}|-L=0 \\
& |\overline{f g}|-D=0
\end{aligned}
$$

where $\overline{m n}$ represents the vector from $m$ to $n$ and $|\overline{m n}|$ is the length of that vector. If $m_{x}$ and $m_{y}$ give the coordinates of the point $m$ then,

$$
|\overline{m n}|=\sqrt{\left(n_{x}-m_{x}\right)^{2}+\left(n_{y}-m_{y}\right)^{2}}
$$

The remaining equations are derived by enforcing synchronization between rolamite pairs. The cross product of the unit vector parallel to a link and the unit vector connecting rolamite pair centers is a measure of the joint angle. This product for each rolamite pair must be equal if the rolamite angles are equal. Figure 7 illustrates these vectors and the following equations result for the three rolamite pairs,

$$
\begin{aligned}
& (\widehat{c d} \times \widehat{c b})_{3}-(\widehat{b c} \times \widehat{b a})_{3}=0 \\
& (\widehat{d c} \times \widehat{d e})_{3}-(\widehat{e d} \times \widehat{e f})_{3}=0 \\
& (\widehat{g h} \times \widehat{g f})_{3}-(\widehat{f g} \times \widehat{f e})_{3}=0
\end{aligned}
$$

where $\widehat{m n}$ indicates the unit vector from $m$ to $n$. These equations are somewhat cumbersome, but they have the beneficial feature of allowing only the physically real solution. The exception is that for angles of both 0 and $\pi$, the cross products of Equation (19) are zero, resulting in identical solutions. In practice, this is not a problem because the false solution is never close to the real solution.

The two-dimensionality of the current problem dictates that the rolamite axes will be parallel by default. Thus, only the $z$ component is used in Equation 19. If the magnitude of the cross products were used, the equations would allow equal and opposite angle false solutions as well. The threedimensional formulation is not so simple as additional equations are required to enforce relative rolamite axis orientations.

The locations of the two chain end links are characterized by the $x$ and $y$ locations of the points $a, b, g$ and $h$. These parameters (eight total) describe the location of the array and bus and are prescribed. Equations (17) and (19) thus represent a set of eight equations with eight unknowns (the $x$ and $y$ locations of the intermediate points $c, d, e$ and $f$ ). The equations were numerically solved using Newton's method in Mathematica 4.0 (Wolfram Research) for a harmonic input displacement amplitude of $5 \mathrm{~mm}(0.20$ in), in both the $x$ and $y$ directions. The excitation amplitude was chosen such that the linear approximation to infinitesimally small vibrations would result. The solution procedure results in a displacement time-history for each point.

D'Alembert's principle is used to dynamically characterize the Traveler-ARM. In equation form, the principle is equivalent to,

$$
\begin{aligned}
& \sum F-m a_{G}=0 \\
& \sum M_{G}-I_{G} \alpha_{G}=0
\end{aligned}
$$

where the acceleration terms represent the d'Alembert forces. $^{10}$ The forces and moments are the internal reaction forces acting at the joint interfaces and are unknown. Solving for them first requires calculation of the link center of gravity linear and angular accelerations. Because the joint location time histories are known, calculating the link center of gravity 
position and orientation time history is straightforward. A numerical second derivative of these quantities with time yields the link accelerations.

The center of gravity of a link is located at the midpoint between the two link end points. For example, a link from $m$ to $n$ has center of gravity location given by,

$$
\begin{aligned}
& P_{m n, x}=\frac{m_{x}+n_{x}}{2} \\
& P_{m n, y}=\frac{m_{y}+n_{y}}{2}
\end{aligned}
$$

Each chain link has a total of four forces acting on it ( $F_{m n, x}$ and $F_{m n, x}$ forces at each rolamite interface, no moment transfer). The forces act at the midpoint between two rolamite pair halves, given by Equation (21). Each link results in three equations: two linear acceleration equations and one angular acceleration equation. Because two links share a common connection point $\left(P_{d e}\right)$, the reaction forces at this location are equal and opposite. A linear set of six equations with six unknown forces results. The unknowns are the six reaction forces at the rolamite interfaces, $F_{b c}, F_{d e}$ and $F_{f g}$ (each force has two components). The components of $F_{d e}$ are considered positive when acting on link $c d$ and directed in the positive $x$ or $y$ directions.

Expanding the translational accelerations of Equation (20) yields,

$$
\begin{aligned}
& F_{b c, x}+F_{d e, x}-m a_{c d, x}=0 \\
& F_{b c, y}+F_{d e, y}-m a_{c d, y}=0 \\
& -F_{d e, x}+F_{f g, x}-m a_{e f, x}=0 \\
& -F_{d e, y}+F_{f g, y}-m a_{e f, y}=0
\end{aligned}
$$

where $m$ is the total mass of a link. Accelerations $\left(a_{m n, x}\right.$ and $a_{m n, y}$ ) are calculated using the classical approach of numerically taking the second derivative of position with respect to time. The discrete time approximation for acceleration is,

$$
a_{m n, x, t}=\frac{1}{\delta^{2}}\left(P_{m n, x, t-\delta}-2 P_{m n, x, t}+P_{m n, x, t+\delta}\right)
$$

where $t$ is the time the equation gives the average acceleration for and $\delta$ is the time between points.

Similarly, two angular acceleration equations can be written by summing the moments about the centers of gravity of links $c d$ and $e f$,

$$
\begin{aligned}
& -F_{b c, x}\left(P_{b c, y}-P_{c d, y}\right)+F_{b c, y}\left(P_{b c, x}-P_{c d, x}\right) \\
& -F_{d e, x}\left(P_{d e, y}-P_{c d, y}\right)+F_{d e, y}\left(P_{d e, x}-P_{c d, x}\right)-I \alpha_{c d}=0 \\
& F_{d e, x}\left(P_{d e, y}-P_{e f, y}\right)-F_{d e, y}\left(P_{d e, x}-P_{e f, x}\right) \\
& -F_{f g, x}\left(P_{f g, y}-P_{e f, y}\right)+F_{f g, y}\left(P_{f g, x}-P_{e f, x}\right)-I \alpha_{e f}=0
\end{aligned}
$$

where $I$ is the rotational inertia of a link. The discrete time equation for angular acceleration of a link is given by,

$$
\alpha_{m n, t}=\frac{1}{\delta^{2}}\left(\theta_{m n, t-\delta}-2 \theta_{m n, t}+\theta_{m n, t+\delta}\right)
$$

Again, the equation gives the average acceleration at time $t$. Equations (22) and (24) form a linear system of six equations with six unknowns. Solving these equations for an acceleration time history calculated with Equations (23) and (25) gives the joint interface force time history.

Transfer functions are calculated by taking the ratio of the output reaction force amplitude to the input displacement amplitude. In the two-dimensional case, 9 transfer functions are available to characterize the vibration isolation qualities of the Traveler-ARM,

$$
\begin{aligned}
& \frac{F_{\max , x}}{\Delta_{\max , x}}, \frac{F_{\max , x}}{\Delta_{\max , y}}, \frac{F_{\max , x}}{\Delta_{\max , \theta}} \\
& \frac{F_{\max , y}}{\Delta_{\max , x}}, \frac{F_{\max , y}}{\Delta_{\max , y}}, \frac{F_{\max , y}}{\Delta_{\max , \theta}} \\
& \frac{M_{\max }}{\Delta_{\max , x}}, \frac{M_{\max }}{\Delta_{\max , y}}, \frac{M_{\max }}{\Delta_{\max , \theta}}
\end{aligned}
$$

In the three-dimensional case, 36 transfer functions are required. The most important transfer functions are expected to be those involving linear motions and forces. Thus, only four of the transfer functions in Equation (26) were evaluated here,

$$
\begin{aligned}
& \frac{F_{\max , x}}{\Delta_{\max , x}}, \frac{F_{\max , x}}{\triangle_{\max , y}} \\
& \frac{F_{\max , y}}{\Delta_{\max , x}}, \frac{F_{\max , y}}{\triangle_{\max , y}}
\end{aligned}
$$

The following table lists geometry and mass assumptions used in the analysis:

\begin{tabular}{|c|c|}
\hline Paramter & Value \\
\hline Link length $(L)$ & $1.52 \mathrm{~m}(60.0 \mathrm{in})$ \\
\hline Rolamite diameter $(D)$ & $10.2 \mathrm{~cm}(4.0 \mathrm{in})$ \\
\hline Link mass $(m)$ & $0.972 \mathrm{~kg}(2.14 \mathrm{lb})$ \\
\hline Link inertia $(I)$ & $0.376 \mathrm{~kg}-\mathrm{m}^{2}\left(1280 \mathrm{lb}-\mathrm{in}^{2}\right)$ \\
\hline
\end{tabular}

The rolamite diameter was chosen based on a reasonable tape thickness and the strain limit of the tape material. The link mass and inertia are derived from 
reasonable assumptions for the sum of rolamite joints, tubes connecting the joints and harnessing.

\section{$\underline{\text { Results }}$}

Even a two-dimensional Traveler-ARM has a large operational area and characterizing its performance requires evaluating the transfer functions over a range of nominal initial positions. Thus, the transfer functions were evaluated at many positions so as to map the Traveler-ARM performance and define the regions of optimal performance and of inoperability. The resulting contour plots are shown in Figures 8 and 9 for $x$ and $y$ reaction forces respectively. In interpreting the plots, consider the system bus to be fixed at the origin. A specific point in the plot corresponds to point $h$ in Figure 6, the SquareRigger connection point. To help illustrate this, multiple Traveler-ARMs are superimposed on the plots. The superimposed Traveler-ARMs also show the intermediate joint locations for a range of operating points.

The contour plots indicate the Traveler-ARM has good vibration isolation characteristics in significant portions of its operational range. Only when mobility range limits are approached do the transfer functions greatly increase. The minimum transfer function values range from 0.000042 to $0.000057 \mathrm{~N} / \mathrm{m}$ with the lower value at $x=1.8 \mathrm{~m}$ and $y=2.2 \mathrm{~m}$ for $F_{\max , y} / \Delta_{\max , y}$ and the greater value at $x=1.0 \mathrm{~m}$ and $y=-2.2 \mathrm{~m}$ for $F_{\max , y} / \Delta_{\max , x}$. It can be seen that the contour lines valued at $0.003 \mathrm{~N} / \mathrm{m}$ encompass a large portion of the operational range.

As indicated in Equation (12), the transfer functions scale with frequency squared (the plots were evaluated for $0.02 \mathrm{~Hz}$ ). Thus, transfer functions at 0.2 $\mathrm{Hz}$ are obtained by multiplying the plots by $(0.2 / 0.02)^{2}$ $=100$.

By knowing the magnitude of the harmonic force acting on a system bus through the Traveler-ARM, it is possible to estimate the resulting jitter error using Equations (5) and (12). As an example, assume a system bus with $6,000 \mathrm{~kg}-\mathrm{m}^{2}$ rotational inertia and $r=$ $1.5 \mathrm{~m}$ (offset from Traveler-ARM connection point to bus center of gravity). Assume a structure vibrates at $0.02 \mathrm{~Hz}$ with amplitude of $2 \mathrm{~cm}$ at the point where it is attached to the system bus through a Traveler-ARM. With a representative Traveler-ARM transfer function value of $0.003 \mathrm{~N} / \mathrm{m}$, the amplitude of the harmonic torque on the system bus would be $0.00009 \mathrm{~N}-\mathrm{m}$ and the resulting jitter error would be $0.95 \mathrm{e}-6 \mathrm{rad}(54 \mathrm{e}-6$ $\mathrm{deg})$. This jitter error is independent of frequency.

\section{Conclusions}

A novel articulated isolator has been presented and characterized for dynamic vibration isolation. The reference design was shown to cause a $0.003 \mathrm{~N}$ harmonic system bus disturbance per meter of isolated structure vibration amplitude. This level of performance was further shown to cause a representative system bus jitter error of only $0.95 \mathrm{e}-6$ rad (54e-6 deg). Structures isolated with articulated isolators were also shown to be much more scalable than fixed structures while maintaining system agility. The isolator disturbance transfer functions were shown to scale with frequency squared, resulting in a system bus jitter error that is independent of frequency. The Traveler-ARM has great potential for enabling new classes of space missions involving large, low frequency space structures. Prime applications include large gossamer structures such as solar arrays, sunshields, solar sails, reflectors and radars.

\section{References}

1 Blevins, Robert D., "Formulas for Natural Frequency and Mode Shape," Krieger Publishing Company, 2001 Reprint Edition

2 Murphy, D. M., Eskenazi, M. I., White, S. F. and Spence, B. R., "Thin-Film and Crystalline Solar Cell Array System Performance Comparisons," 29th IEEE PhotoVoltaic Specialists Conference, New Orleans, May 22, 2002

3 Botke, M., Murphy, D., Murphey, T., Sorensen, P., "Zero Deadband, Multiple Strut Synchronized Hinge for Deployable Structures," Proceedings of the $36^{\text {th }}$ Aerospace Mechanisms Symposium, May 2002, NASA /CP-2002-211506

4 Meink, T., Reinhardt, K., Luu, K., Blankenship, R., Huybrechts, S., Das, A., "Powersail - A High Power Solution," AIAA Space 2000 Conference and Exposition, Long Beach, CA, Sept. 19-21, 2000

5 Young, W. C., "Roark's Formulas for Stress \& Strain," 6 ${ }^{\text {th }}$ Ed., McGraw-Hill, Inc., 1989

6 Brown, G. W., "Equipment for use with hand held motion picture cameras" U.S. Patent Number $4,017,168$

7 The Tiffen Commpany, LLC (www.steadicam.com)

8 Wilkes, D. F., "Roller-Band Devices," U.S. Patent Number 3,452,175

9 Brush Wellman Company (www.brushwellman.com)

10 Waldron, K. J. and Kinzel, G. L., "Kinematics, Dynamics, and Design of Machinery," John Wiley \& Sons, Inc., 1999 

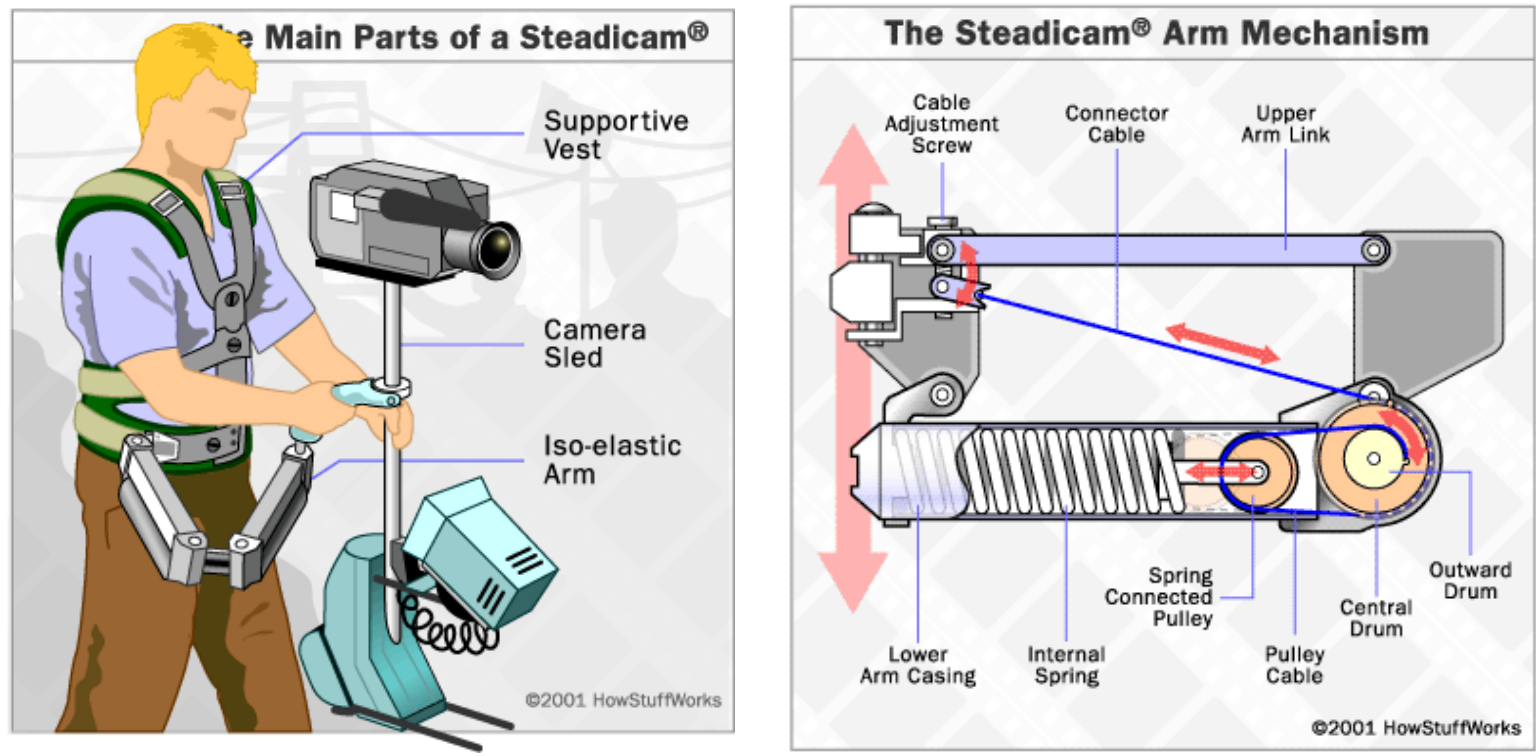

Figure 1: Steadicam parts and mechanical operation (The Tiffen Company, LLC).

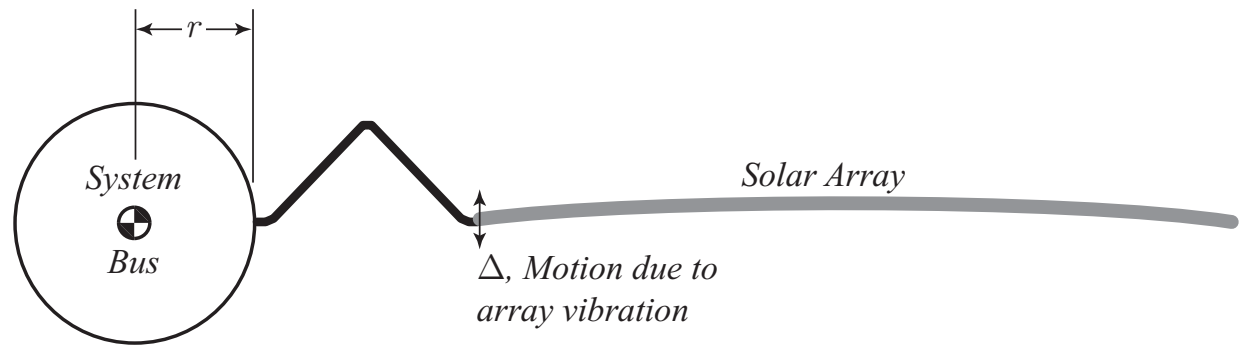

Figure 2: Schematic diagram of simplified articulated isolator system model.

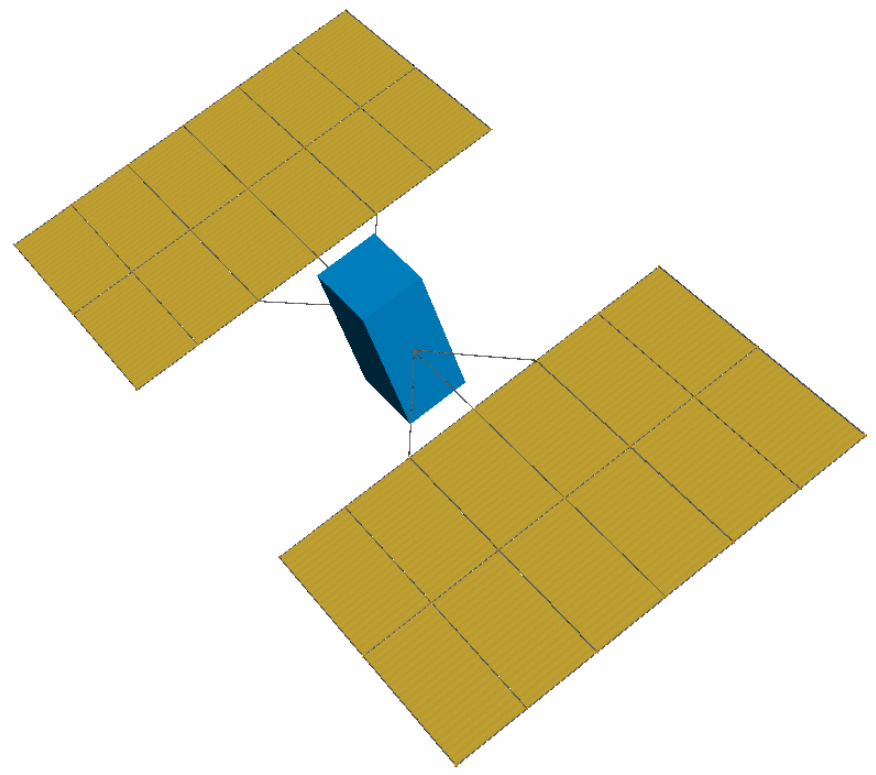

Figure 3: SquareRigger in a traditional wing configuration (Traveler-ARMs not shown). 


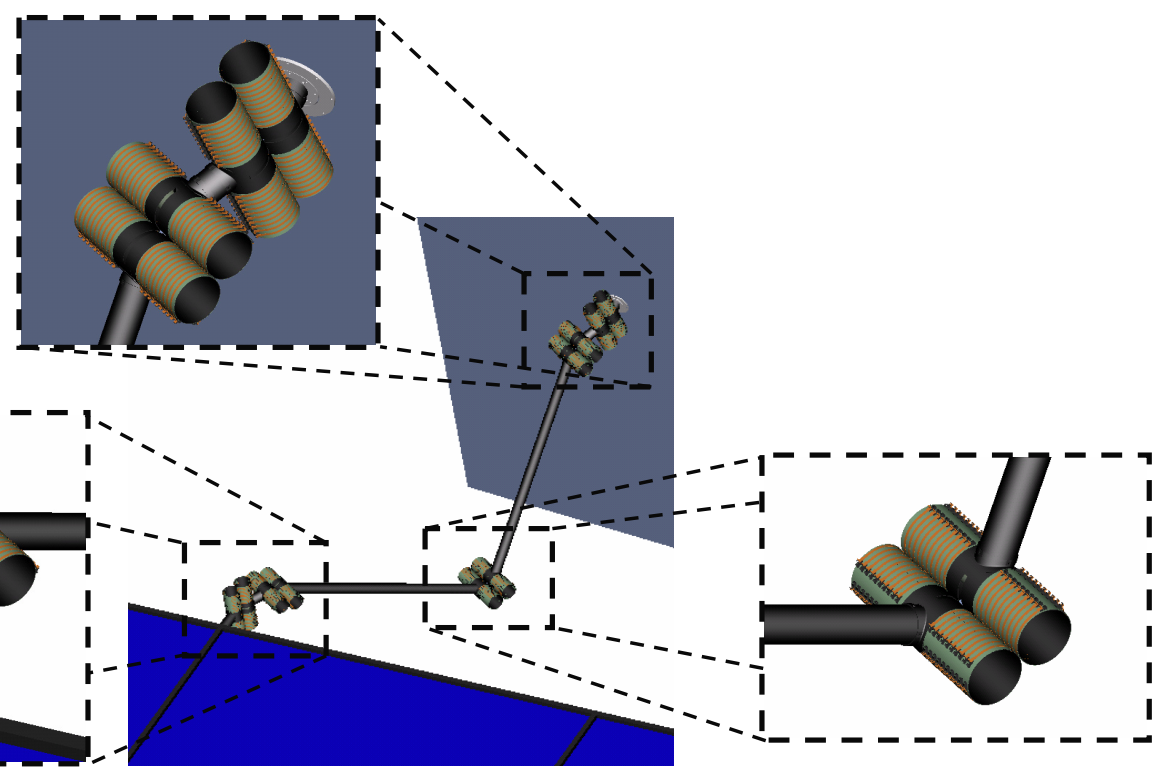

Figure 4: Traveler-ARM rolamite joint locations and orientations.

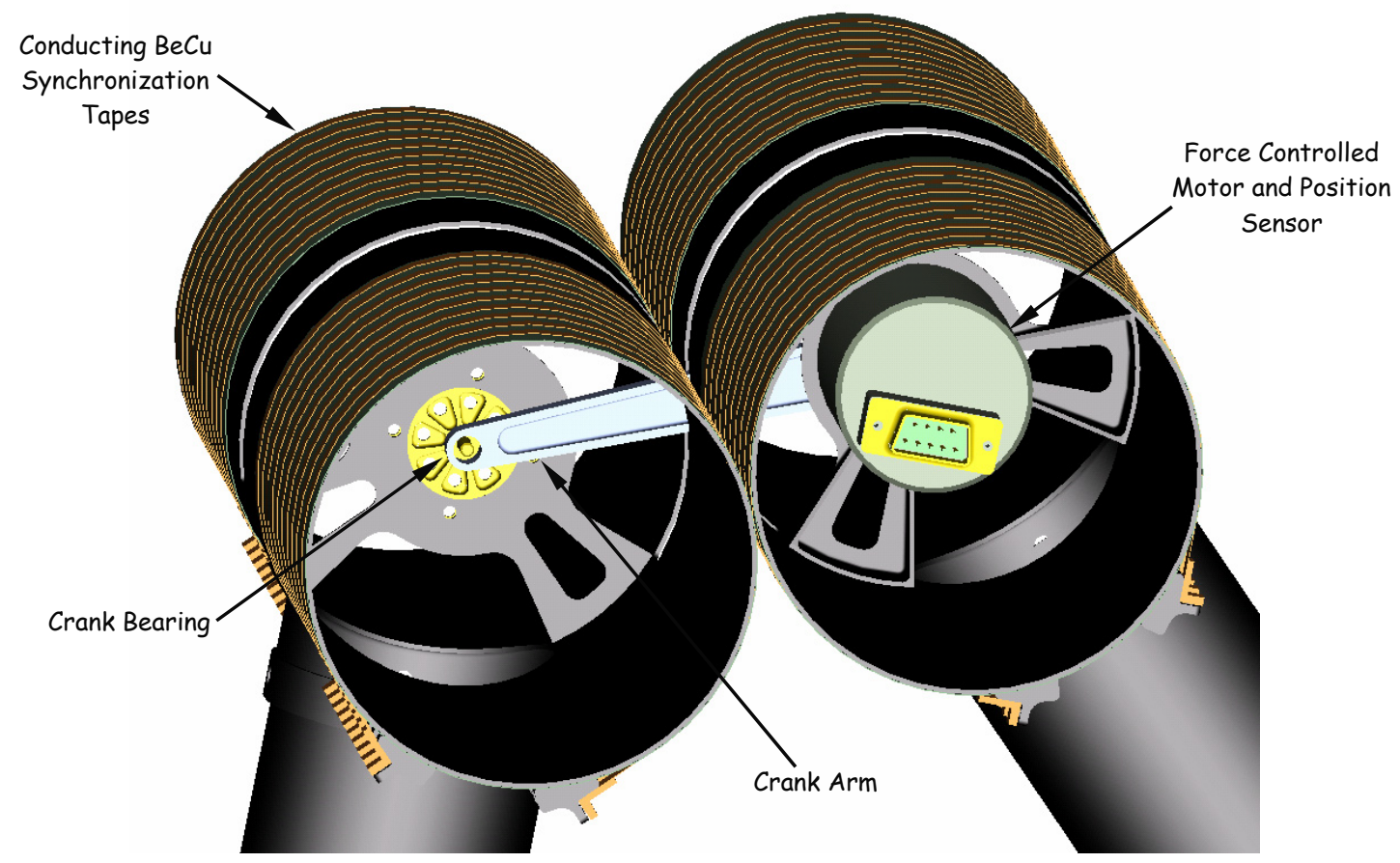

Figure 5: A force controlled, conducting rolamite joint. 


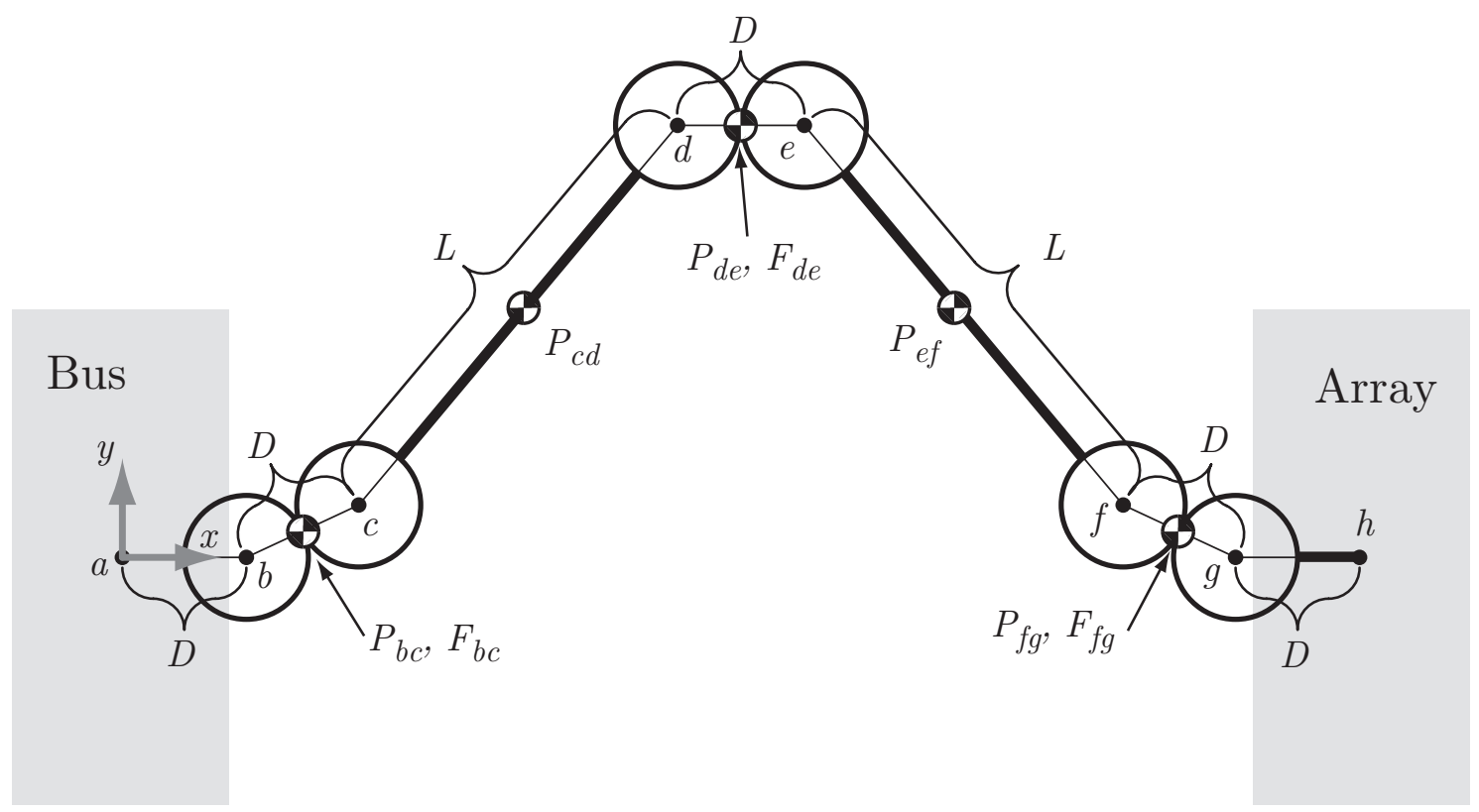

Figure 6: Schematic diagram of a two-dimensional Traveler-ARM.

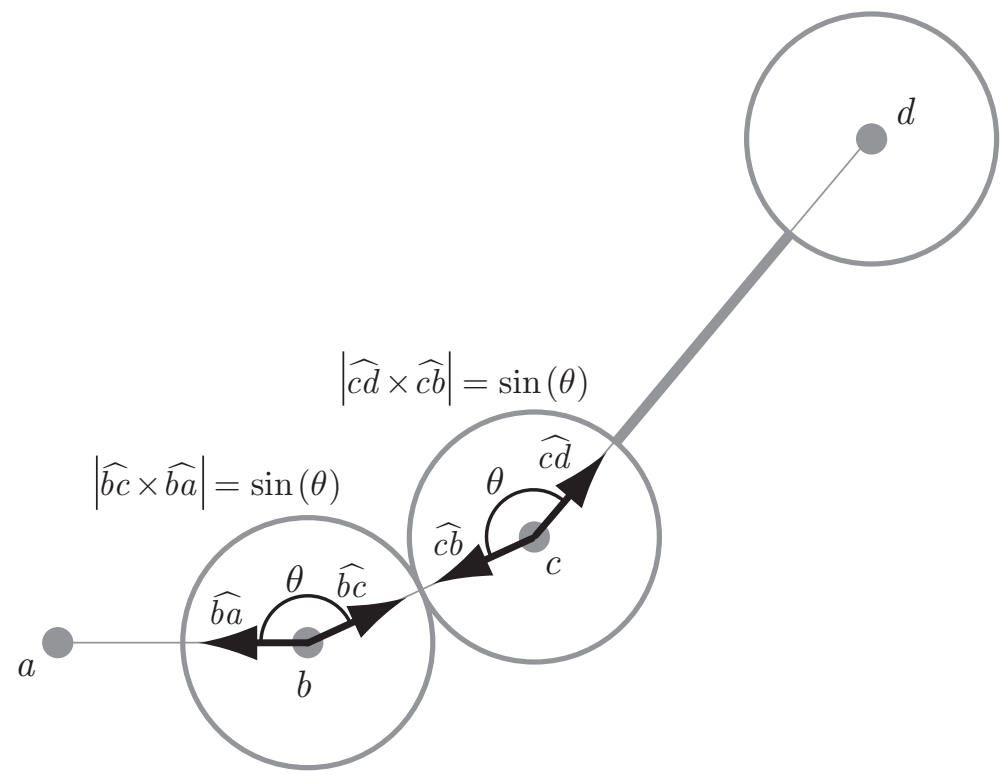

Figure 7: Rolamite equal angle cross product definition for kinematic equations. 

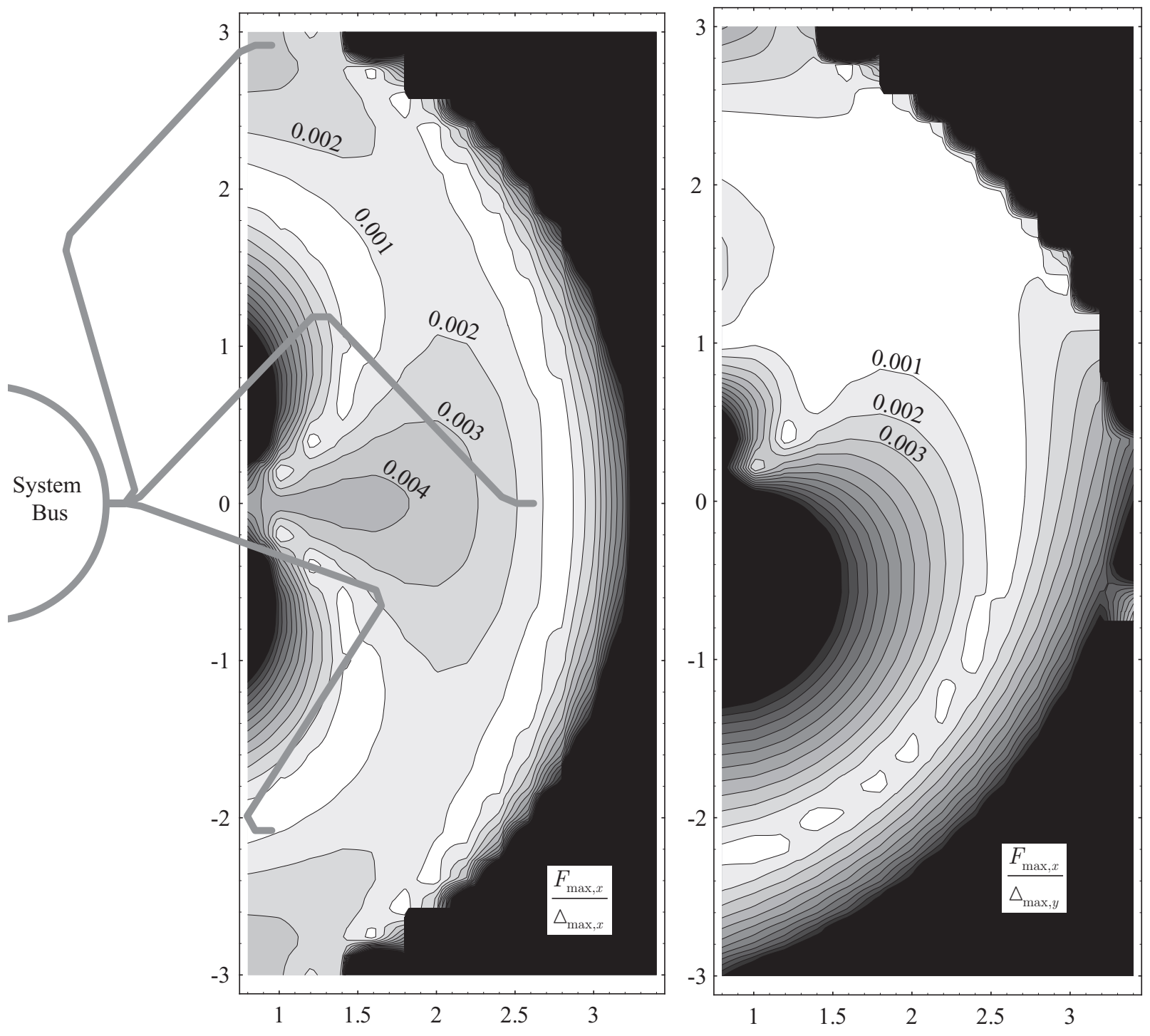

Figure 8: Reference Traveler-ARM reaction force transfer functions for $\frac{F_{\max , x}}{\Delta_{\max , x}}$ and $\frac{F_{\max , x}}{\Delta_{\max , y}}(\mathbf{N} / \mathbf{m}, \mathbf{0 . 0 2} \mathbf{H z})$. 

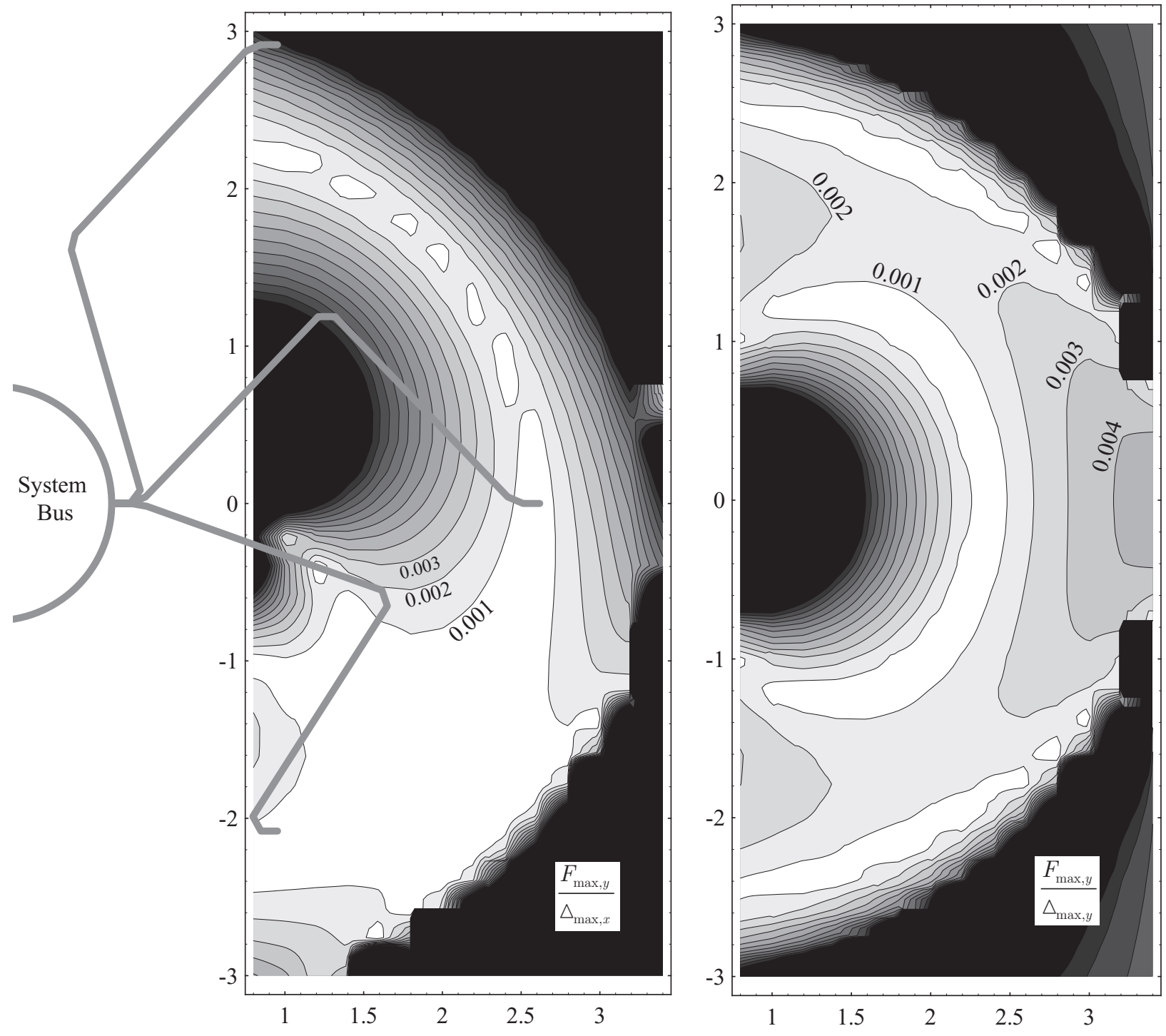

Figure 9: Reference Traveler-ARM reaction force transfer functions for $\frac{F_{\max , y}}{\triangle_{\max , x}}$ and $\frac{F_{\max , y}}{\triangle_{\max , y}}(\mathbf{N} / \mathbf{m}$, at $\mathbf{0 . 0 2} \mathbf{H z})$. 